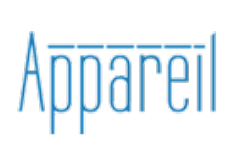

Appareil

4 | 2010

Biopolitique, éthique et subjectivation, questions de modernité

\title{
Régimes de duplicité, espaces désesthétisés
}

\section{Christiane Vollaire}

\section{(2) OpenEdition \\ Journals}

Édition électronique

URL : https://journals.openedition.org/appareil/899

DOI : 10.4000/appareil.899

ISSN : 2101-0714

Éditeur

MSH Paris Nord

Référence électronique

Christiane Vollaire, "Régimes de duplicité, espaces désesthétisés », Appareil [En ligne], 4 | 2010, mis en ligne le 27 janvier 2010, consulté le 12 octobre 2021. URL : http://journals.openedition.org/appareil/ 899 ; DOI : https://doi.org/10.4000/appareil.899

Ce document a été généré automatiquement le 12 octobre 2021.

\section{(c)}

Appareil est mis à disposition selon les termes de la Licence Creative Commons Attribution - Pas d'Utilisation Commerciale - Pas de Modification 4.0 International. 


\title{
Régimes de duplicité, espaces désesthétisés
}

\author{
Christiane Vollaire
}

1 À l'origine de ce travail, il y a un constat: celui des formes affolantes que prend la déraison politique quand elle se présente sous le masque de la rationalité. Et la problématique actuelle des migrations nous paraît au cœur de ce constat, dans toute la puissance de son ambivalence.

2 Cette ambivalence tient d'abord à la question centrale du partage territorial, liée à celle de la visibilité des existences. C'est donc en termes d'espace qu'elle se pose, et c'est sous cette forme qu'on ne cesse de la rencontrer.

3 Cette rencontre se présentera en particulier, ici, sous la forme de ce qu'on pourrait appeler, par quelque chose qui ressemble beaucoup à un oxymore, un travail philosophique de terrain.

\section{Rendre visible}

4 Il a pris naissance dans l'été 2008 en Pologne, et s'est élaboré à partir des entretiens effectués auprès de 104 personnes réfugiées, en majorité tchétchènes, dans 16 centres d'hébergement ouverts et 2 centres de rétention fermés; et auprès de 35 responsables polonais d'ONG, d'OIG et des organismes administratifs polonais en charge des réfugiés.

$5 \quad$ L'ouvrage qui se prépare à partir de là met en interaction un travail photographique fait dans ces lieux d'hébergement et de rétention, et un travail philosophique élaboré à partir des entretiens avec les personnes qui y vivent. Les photographies ne montrent pas les personnes, le texte ne présente pas les lieux. Mais la parole des personnes est marquée par les lieux de leur assignation, comme les lieux, vides sur les images, sont marqués par la présence des gens. Et c'est de cette co-présence, ou de ce que le sociologue Abdelmalek Sayad aurait appelé une «double absence », que ce travail est tissé. 
6 Les entretiens, informels, obtenus au hasard des rencontres dans un couloir, au détour d'une allée, sur le pas de porte d'une chambre, dans l'espace commun d'un hall, d'une salle de classe ou d'une cuisine collective, ont été bien sûr sollicités mais jamais arrachés. Mes interlocuteurs voulaient parler, et certains même se sont battus contre les barrières de la langue, parfois contre celles qu'opposaient certains responsables de centres; parfois aussi contre celles du regard des autres, pour entrer en communication. Tous savaient que nous ne pouvions rien pour eux, que nous n'étions membres d'aucune ONG et n'avions aucun pouvoir administratif ou décisionnel. Mais tous savaient aussi que ce livre allait exister, qu'il transmettrait leur parole et montrerait leurs lieux de vie en transit. Et tous voulaient que cela soit su.

7 Ce désir de visibilité, ce désir d'apparaître dans l'espace public, est l'objet philosophique de cet ouvrage. Car la vie des demandeurs d'asile est d'abord une vie marquée par la clandestinité, une vie soustraite au regard commun dans la mesure même où elle est exposée au contrôle. Une vie toujours offerte au double langage, à la duplicité des pouvoirs politiques sur leurs terrains d'origine où ils sont à la fois offerts à la violence et assignés à demeurer; mais aussi sur leurs terrains "d'accueil ", où ils sont répertoriés en vue de l'expulsion ; fichés pour pouvoir être non pas intégrés, mais éjectés. Ou, au mieux, autorisés à résider mais sans pouvoir accéder ni au logement ni au travail. Et ce jeu du visible et de l'invisible dépasse ici, dans tous les sens du terme, les limites du terrifiant.

8 Les personnes que nous avons rencontrées constituaient évidemment une élite : celle que constituent tous les réfugiés qui ont réussi à survivre à la violence de leurs dirigeants, à celle de la police, à celle des passeurs, à celle de leurs compagnons de route, à celle des infiltrés de leurs lieux d'origine qui continuent souvent à les poursuivre, à celle parfois de leur propre famille, à celle de la promiscuité, à celle des maffias omniprésentes, qui infiltrent aussi bien les pays d'origine que les pays d'accueil, et sont aussi présentes dans les centres d'hébergement. Car le sans-droit est celui qui, parce qu'il n'est pas protégé par les pouvoirs légitimes, est le plus exposé aux pouvoirs occultes. Et les pouvoirs politiques qui refusent le droit d'asile abandonnent très sciemment les demandeurs à cette violence-là.

9 Mais cette élite de la survie est elle-même extrêmement diverse dans ses représentants, issus d'une grande variété de milieux sociaux et destinés à cohabiter dans les mêmes centres.

10 Vouloir rationaliser les processus migratoires devrait en effet d'abord revenir à en analyser l'origine. Or les politiques mises en place aussi bien aux USA qu'en Europe font précisément l'économie de cette analyse. Que le meilleur moyen d'éviter la fuite des populations soit de leur garantir la possibilité de vivre sur leur propre territoire, ou du moins d'en favoriser la possibilité, ne semble effleurer ni les organisations internationales, ni les puissances économico-politiques, qui continuent à admettre ou à produire le pillage des ressources et la corruption des élites, dans le temps même où elles interviennent policièrement sur la migration des populations.

\section{Clandestinité et violence de l'occulte}

11 Vouloir rationaliser les processus migratoires devrait aussi revenir à analyser les conséquences de la contrainte policière à la clandestinité : des sujets voués à se cacher 
sont nécessairement voués à recourir à des pouvoirs occultes et parallèles, et ils sont de ce fait livrés à eux. Dans Gomorra, Roberto Saviano montre avec une imparable rigueur comment les pouvoirs maffieux se nourrissent de la clandestinité. Et l'association La Strada met en évidence la relation entre migration clandestine, prostitution et trafics d'organes. Tout sujet privé de la protection des lois est nécessairement offert et délibérément exposé à la violence des maffias. Les lois qui bloquent massivement les migrations provoquent donc l'expansion quasi-mécanique des systèmes maffieux, qu'elles contribuent à nourrir.

C'est la décision maffieuse qui assumera cette injonction paradoxale: jouant de l'interdit pour faire augmenter la demande, elle assure la diffusion par une banalisation des circuits intermédiaires.

Ici, c'est la modalité postmoderne de l'ultralibéralisme qui conditionne l'efficacité de l'intervention maffieuse. Ayant montré la somme d'investissement économique que suppose la production des toxiques ou la circulation des corps, Saviano montre tout ce que le sujet, comme produit commercial, doit à une planification méticuleuse et très professionnelle de sa distribution. Et c'est dans cette planification qu'entre la stratégie de banalisation. Celle-ci ne vise pas à permettre l'autorisation, mais la désinhibition des sujets quant à l'interdit : il faut que la transgression ne nécessite aucune complication. Et pour cela, il faut que son caractère délictueux soit d'une certaine façon occulté.

Saviano montre comment l'économie maffieuse est une économie de marché qui a parfaitement intégré les règles de la promotion industrielle de la marchandise, et produit ainsi une vaste circulation mondialisée des corps et des choses; des corps devenus choses, fondus dans la circulation des fluides vitaux, informatiques et financiers. Une indifférenciation qui rend indistincte la frontière entre l'intériorité des corps et l'extériorité des choses ; mais aussi entre l'intériorité mentale et l'extériorité physique. Le livre s'ouvre dans le port de Naples, sur l'image d'un container suspendu et tout à coup lâché, dont la rupture au sol s'ouvre sur des dizaines de cadavres de Chinois clandestins, dont même le rapatriement des corps ne peut pas être officialisé.

En prétendant donc parer un danger fictif, c'est un danger réel que l'on provoque. Danger qui ne menace pas seulement les clandestins, mais le corps social tout entier.

Partout où le phénomène des migrations est refoulé, le processus même de son refoulement produit un effet dans le réel et dans le symbolique. Dans le réel, c'est un effet immédiatement meurtrier: des milliers de gens meurent tous les jours non pas des effets de la violence des pays qu'ils ont fui, mais des effets de la violence policière des pays qui leur refusent l'accueil. Et meurent aussi, physiquement ou mentalement, de la violence des puissances occultes auxquelles ils sont livrés, qui se partagent leurs corps et vendent leur sexualité comme une marchandise. Dans le symbolique, c'est un effet destructeur. Ce n'est pas seulement l'idée d'humanité qui est ici clivée, mais c'est la possibilité même pour des sujets citoyens de se reconnaître une appartenance à des pays qui refusent l'accueil et condamnent délibérément à l'indignité.

17 Dès lors, la limite même du visible et de l'invisible, du dicible et de l'indicible, devient indéterminable, contraignant les sujets à s'absorber dans le devenir des choses. 


\section{Un processus de liquidation}

18 C'est de cette absorption, liée à une nouvelle forme de la modernité, que traite Zygmunt Bauman en forgeant le concept de « vie liquide ». Il écrit ainsi dans Le Présent liquide :

La modernité est en train de passer de la phase solide à une phase liquide, dans laquelle les formes sociales (les structures qui limitent les choix individuels, les institutions qui veillent au maintien des traditions, les modes de comportement acceptables) ne peuvent plus - et ne sont plus censés - se maintenir durablement en l'état, parce qu'elles se décomposent en moins de temps qu'il ne leur en faut pour être forgées et se solidifier ${ }^{1}$.

Opérer cette analyse, c'est précisément retourner le modèle cartésien contre luimême: là où la modernité intellectuelle affirmait, contre les tyrannies religieuses auxquelles elle s'affrontait, les exigences rationnelles de la clarté et de la distinction, un certain concept de la modernité économique, devenu prépondérant, a fini par produire les formes déstructurantes de l'indistinction; et, au sens propre, une liquidation des idéaux prétendus de la modernité.

20 C'est cette liquidation qui, fluidifiant les circuits, fluidifie aussi les sujets, et produit cette fusion du corps dans l'espace indéterminé de la circulation des choses. Et ce modèle fusionnel du développement industriel est précisément celui du processus de globalisation. Bauman, dans S'acheter une vie, paru en 2008, établit le rapport de cette liquidation aux liquidités de l'économie de marché en analysant les devenirs actuels de ce qu'on appelle "société de consommation». Il y écrit ainsi que «la remarchandisation constante est à la marchandise, et donc au consommateur, ce que le métabolisme est aux organismes vivants ». Cette analogie entre économique et organique montre en quoi les dérégulations collectives induisent les dérégulations individuelles.

21 Mais elle permet aussi d'éclairer en quoi l'économie de l'informatique fonctionne nécessairement comme liquidation des frontières entre espace privé et espace public, entre intériorité mentale et extériorité économique, entre imaginaire et réel, imposant le virtuel, et l'indifférenciation à l'égard du devenir des corps, comme mode de rapport à l'existence.

22 Ainsi des processus de désesthétisation sont-ils liés à cette contradiction radicale : un effacement des frontières dans la globalisation des circuits financiers et des réseaux de communication, dans le temps même d'une intensification des frontières comme obstacles à la circulation des personnes. La modernité des spatialisations économiques s'y affronte à un archaïsme des représentations de l'espace national.

\section{Formes affolantes de l'irrationalité politique}

23 Ce qui sera mis ici en évidence est donc d'abord la forme de folie induite par une prétendue raison politique. À tous les niveaux de nos rencontres, de nos entretiens et de notre réflexion apparaît cette forme destructrice de l'irrationalité politique se présentant sous les oripeaux de la raison d'État. Et c'est cet oxymore d'une rationalité radicalement irrationnelle qui nous a concrètement saisis ici. 

structuralisme:

Je n'admets absolument pas l'identification de la raison avec l'ensemble des formes de rationalité qui ont pu, à un moment donné, à notre époque et tout récemment encore, être dominantes dans les types de savoir, les formes de technique et les modalités de gouvernement ou de domination, domaines où se font les applications majeures de la rationalité 2 .

C'est tous les jours et dans les propos et les comportements de chacun que nous avons pu vérifier la pertinence de cette analyse. Les formes de rationalité mises en œuvre par les systèmes politiques contemporains à l'égard des migrations sont tout sauf une manifestation de la raison.

Et les réfugiés ne dénonçaient pas seulement la folie directement criminelle et meurtrière des pays qu'ils avaient fui, mais la folie administrative, différemment destructrice, des pays où ils demandaient à vivre. "Ce système rend fou " était une phrase qu'on entendait souvent. Et il n'y avait effectivement aucun sens à l'errance circulaire qu'on leur imposait sans fin d'un refus d'asile à un autre. Comme le dit l'un des demandeurs d'asile interrogés :

En Tchétchénie, vous n'avez aucune chance de vivre normalement pour des raisons physiques : c'est dangereux. Ici, c'est plus en sécurité, mais le danger est psychique ou moral, et il peut vous détruire.

Mais ce système rendait aussi fous ses propres responsables : responsables des centres qui ne savaient comment répondre à des demandes de soin, d'amélioration de l'hébergement, de sécurité dont ils étaient supposés avoir la charge. Médecins ou infirmiers qui retournaient leur impuissance en indifférence; administrateurs qui ne parvenaient pas à donner les informations juridiques qu'on leur réclamait, tant elles étaient contradictoires; garde-frontières qui laissaient apparaitre la honte qu'ils éprouvaient à sanctionner et à enfermer des sujets qui n'avaient pas commis d'autre délit que celui de vouloir survivre.

De cette honte et de ce non-sens des directives européennes, de cette folie administrative de la décision juridique et politique, témoignait la duplicité des régimes de langage à laquelle les réfugiés étaient en permanence affrontés. Les lois supposées protectrices étaient précisément celles qui les exposaient au danger en les livrant au non-droit et au non-lieu.

Mais ce non-lieu est aussi ce qu'on peut appeler un régime de désesthétisation: empêcher des sujets d'habiter, ce n'est pas seulement les empêcher d'assurer leur survie physique, mais aussi leur interdire de se représenter à eux-mêmes, d'avoir part à l'espace commun qui permet de s'identifier comme humain, ce que montrait Hannah Arendt, dans son essai sur l'impérialisme, à propos des sans-droit.

Les sollicitations à la parole, effectuées dans les centres de réfugiés en Pologne, mettent alors en évidence les différents niveaux de ce régime de duplicité, inscrivant le biopolitique non pas seulement dans la perspective classiquement foucaldienne d'un régime de contrôle, mais dans les contradictions internes d'un contrôle accru et d'une dérégulation. Sans cesse, les migrants sont confrontés à cette duplicité juridique et politique qui fait de la "régulation des flux» une pure et simple amorce de la disparition des personnes. Les régimes de duplicité contemporains, en usant du double langage juridique pour euphémiser la réalité de la violence, sont donc au fondement d'une véritable perversion du politique. 
31 Et cette duplicité mondialisée s'avère, pour les migrants, véritablement terrorisante : livrant les sujets au double langage de l'abandon et de la protection, elle laisse en suspens au-dessus d'eux une menace, implicite et au sens propre affolante, d'extermination.

\section{Régimes de vérité et scénographie de l'accueil}

Pourtant, ce qui nous a saisis, dans ce maelström de la disparition du politique, c'est aussi une réelle capacité d'affronter la déréliction, de dénoncer le double langage et de se situer sur le terrain non pas de l'attentisme, mais de la revendication. Nos interlocuteurs voulaient nous parler, mais ils voulaient aussi faire face aux injonctions paradoxales des pouvoirs dont ils dépendaient, autant qu'aux menaces de disparition physique ou politique qui pesaient sur eux.

Mes questions étaient totalement élémentaires : Pourquoi êtes-vous parti ? Où voulezvous aller? Comment vivez-vous ici ? Et puis celle-ci : Quelle image positive gardezvous de votre lieu d'origine? Mais, à mon grand étonnement, aucune de ces questions très simples ne leur avait jamais été posée sous cette forme-là. Et tous me disaient parler ici pour la première fois de ce sur quoi ils n'avaient jamais été interrogés. Du moins n'avaient-ils pas l'impression d'avoir été interrogés en vue d'ouvrir un espace d'attention, mais seulement formellement questionnés en vue de fournir des informations. En quelque sorte, mis à la question mais jamais sollicités à la parole.

Mes raisons pour le faire n'étaient nullement désintéressées : je voulais comprendre, et je voulais écrire. Mais il se trouvait que nos intérêts coïncidaient, et de cette coïncidence est souvent (évidemment pas toujours) né quelque chose d'une étrange relation de confiance et de soulagement, dans un contexte qui ne s'y prêtait guère. Des relations, parfois commencées sous le signe d'une légitime défiance, ou même d'une hostilité, se sont souvent dénouées.

Dans tous les cas, le régime de l'entretien n'est pas un régime de vérité. Et la façon dont s'articulent sincérité, authenticité et véridicité, déjà difficile à tous les niveaux d'une existence relativement simple, devient, dans des situations aussi limites que celles auxquelles on est affronté ici, particulièrement complexe. Je n'ai donc en aucun cas recherché ni mis en place ce que Foucault appelait le régime de l'aveu. Je ne demandais à personne de prêter serment, mais seulement de me parler. Et les convergences de leurs discours étaient déjà suffisamment éclairantes. Bien des choses m'ont évidemment été cachées, et le désir de visibilité de ces personnes ne me donnait nullement ni le droit ni l'accès à leur intimité, ou à ce qu'elles estimaient nécessaire de ne pas dire. Et il est arrivé que mon interprète, très attentive, refuse à juste titre en certains contextes de traduire certaines questions.

Quelque chose de l'ordre d'une scénographie se nouait toujours autour de ces entretiens, et celle-ci était souvent aussi éclairante que la parole elle-même. L'apparition ou la disparition de certains intervenants, les silences ou les regards de certains autres, leur mode d'intervention dans le dialogue, la manière dont une collation était amenée, dont une table était disposée ou une chaise avancée, me disaient qu'en ces lieux-là nous étions les invités de ceux qui n'étaient invités nulle part. Et ces lieux de relégation, par un effet d'aberration de l'espace-temps, s'ouvraient à chaque 
fois sur l'immensité d'un nouvel espace : celui d'un projet d'existence dont tout, dans nos dispositifs juridiques, vise à obstruer la réalisation.

\section{L'esthétique comme arrachement au biologique}

Dans Ce qui reste d'Auschwitz, Agamben pose la question :

Existe-t-il une humanité en l'homme, que l'on puisse distinguer et séparer de son humanité biologique?

Si Foucault donne une réponse positive à cette question, c'est en opposant, par le biais même du corps, l'humanité biologique à l'humanité esthétique: si les processus de subjectivation passent par le corps, c'est nécessairement par une corporéité distanciée de sa stricte organicité, et en quelque sorte désaffiliée de l'assujettissement biologique à la passivité sociale. Or cette désaffiliation est précisément son esthétisation.

C'est cette relation tensionnelle entre biologique et esthétique, qu'interrogeait en 1790 la Critique de la faculté de juger de Kant, quarante ans après la parution de l'Aesthetica de Baumgarten. Les deux parties de l'ouvrage, faculté de juger esthétique et faculté de juger téléologique, interrogent en effet d'une part la finalité du jugement de goût à travers une représentation de l'homme comme sujet de l'émotion esthétique, et la finalité de la nature à travers une représentation de l'homme comme organisme biologique. Et l'on pourrait dire à partir de là que l'ouvrage n'est rien d'autre qu'une anthropologie, en se souvenant que Foucault a été le traducteur, en 1964, de L'Anthropologie au point de vue pragmatique de Kant, et a publié en 1984 une analyse de Qu'est-ce que les Lumières ?, qui pose la question permanente de la présence du penseur à l'actualité de son temps.

Or pour Kant, comprendre l'homme comme être biologique, c'est d'abord l'inscrire dans la finalité esthétique d'une représentation de soi qui l'arrache, précisément, au biologique. En termes kantiens, il s'agit d'articuler la question de la nature à celle de la liberté, ou de penser la tension entre une existence biologique dans son individuation uniformisante, et une existence politique dans sa subjectivation relationnelle, comme Arendt le mettra en évidence dans son commentaire de la Critique de la faculté de juger. Le jugement de goût est ce qui manifeste en l'homme cette capacité de subjectivation, en ce qu'il le relie à ce que Rancière appellera un "partage du sensible», la faculté corrélative d'éprouver intimement des émotions et de les communiquer, dans le contexte même d'une partition de l'espace politique.

Le sujet émotionnel est un sujet sensible, qui vibre par son corps et par ses sens, mais pour qui précisément cette vibration désigne un au-delà du repli sur la physiologie. Et en ce sens, ce que Kant appelle supra-sensible n'est rien d'autre que ce qui nécessite la médiation sensible, et le partage d'une corporéité non élémentairement organique. Quelque chose de cette intuition résonne encore dans ce que Deleuze et Guattari, écrivant Mille Plateaux, appelleront, à la suite d'Artaud, le « corps sans organe », corps par excellence vibratile et nerveusement réactif à l'invasion émotionnelle, au-delà de la simple contiguïté organique. Corps surtout actif, au-delà de la simple passivité proprioceptive de son système neurobiologique.

42 Partout, dans le déroulement des entretiens comme dans les espaces où ils s'inscrivaient, nous est apparu ce phénomène constant d'une volonté de réesthétisation: réappropriation des lieux indifférenciés de la relégation par la 
disposition des objets qui les habitaient ; manière de poser une nappe, de punaiser une image au mur, d'installer une couverture, d'amener une collation, de servir une boisson. Manière aussi de choisir ses mots, de dresser son corps, de poser son regard. De choisir la parole contre l'indétermination du silence, ou l'éloquence du silence contre la spontanéité de la parole.

\section{Enjeux de subjectivation du biopolitique}

43 Cette esthétique de l'existence s'affirme en quelque sorte comme un contrebiopouvoir: à la discipline comme "gouvernement des vivants» produisant l'assujettissement, Foucault oppose l'ascèse comme "gouvernement de soi », processus de subjectivation permettant d'«élaborer sa vie comme une œuvre d'art». Cette opposition entre l'ascèse autonomisante et la discipline hétéronomique, se voit ainsi sans cesse inscrite au cœur d'un processus de résistance.

C'est cette dualité ascèse/discipline, recouvrant la dualité subjectivation/ assujettissement, qui place la problématique esthétique au cœur d'un questionnement biopolitique. Foucault affirme très clairement, à l'encontre des positions idéalistes, qu'il n'y a pas de naturalité originelle du sujet, et pas davantage de consistance d'un sujet volontairement construit. Mais il affirme tout aussi clairement qu'il n'y a pas pour autant disparition du concept de sujet. La subjectivation est l'objet d'une construction sociale, d'un processus d'élaboration sans fin ; mais ce processus d'élaboration, tel qu'il est politiquement organisé, est lui-même dissolvant : l'individu en subjectivation est sans cesse menacé d'être détruit par cela même qui lui donne les moyens de sa construction.

Cette menace est mise en évidence par la fonction même du langage, qui a cette double puissance de normer et de singulariser. Dans les Problèmes de linguistique générale, publiés en 1966, Benveniste mettait en évidence cette polyvalence paradoxale de la langue. Et il n'est pas indifférent que la mouvance qu'on a dénommée « structuraliste » (et dans l'orbite de laquelle on a situé, à son corps défendant, Foucault) se soit dessinée autour de ses travaux. Dans Ce qui reste d'Auschwitz, Agamben établit du reste un parallèle intriguant autour de l'année 1969, entre la parution de L'Archéologie du savoir de Foucault, et l'aphasie qui s'empare de Benveniste trois ans avant sa mort, affirmant qu' "un fil secret lie son programme à celui du linguiste», autour de la fonction du « Je » comme instance d'énonciation.

Le référent normatif est, pourrait-on dire le vecteur d'un processus de cristallisation de soi, mais il constitue aussi le danger d'une dissolution de soi dans l'hétéronomie. La subjectivation ne peut ainsi échapper à la dissolution que comme résistance, force dynamique s'exerçant à l'encontre du pouvoir qui la produit, du sein même de ce pouvoir. Ce que dit le concept de biopolitique, c'est qu'à aucun moment l'élaboration subjective n'échappe au pouvoir, parce que le pouvoir ne s'affirme jamais comme transcendant, mais toujours comme immanent: la puissance de résistance est puissance à la fois transgressive et constituée sur ce qu'elle transgresse.

Dans les lieux du refuge comme dans ceux de la rétention, l'immanence du biopouvoir est ainsi son omniprésence: le fait qu'il ne s'affirme pas comme principe de souveraineté, mais comme principe de diffusion. Et Foucault montre que cette diffusion produit aussi une indétermination: la diffusion normative du biopouvoir dans les instances individuelles produit aussi une diffusion des corps individuels dans le régime 
de la population, qui n'est rien d'autre qu'une réduction du corps à l'organique : réduction des individus à se fondre comme parties d'un tout, à s'identifier comme désingularisés.

La réduction à l'organique n'est pas seulement réduction d'un individu à sa physiologie; elle est aussi réduction des individus à se penser comme organes d'une totalité. C'est cette double réduction que produit le biopouvoir par sa puissance biologique de diffusion. Et, dans le cas des demandeurs d'asile, cette réduction est d'autant plus perverse qu'elle est discriminante au lieu d'être intégrante. La population des "déplacés» est celle à laquelle le pouvoir de contrôle n'assigne pas même l'emplacement protecteur.

\section{Ambivalence de l'invisibilité}

L'humain réduit à ses fonctions biologiques paraît ainsi un humain dont la corporéité organique a envahi tous les champs de subjectivation, et qui ne peut donc plus se singulariser: toute subjectivation politique est niée par l'individualité organique au double sens du terme, comme à la fois constituée d'organes, et instrument de pouvoir.

50 Tocqueville, dans La Démocratie en Amérique, avait déjà montré que l'individualisation est massifiante, précisément parce qu'elle produit une sorte d'animalisation par le besoin. Et Arendt, dans La Condition de l'homme moderne, avait déterminé les trois niveaux de l'existence humaine (travail, œuvre et action) en montrant que les sociétés contemporaines tendaient à absorber le troisième niveau dans le second: l'action politique tend à se réduire à la production des biens par une absorption du politique dans l'économique. Mais le second niveau lui-même tend aussi à s'absorber dans le premier: la production elle-même tend à se réduire à la réponse au besoin physiologique, à la subsistance biologique d'individus désubjectivés.

51 Foucault reconnaît bien dans le biopolitique cette double réduction indifférenciante. Mais il complexifie ces mécanismes d'indifférenciation assujettissante et de massification normative, en montrant qu'ils deviennent aussi, dans le même temps, les moyens de transgression de la norme. Or ces moyens de transgression ne sont nulle part ailleurs que dans la possibilité de se représenter à soi-même autrement que comme fonction organique ou comme visibilité régularisable.

52 L'opposition ascèse / discipline devient ainsi l'opposition esthétique / visibilité. Et dans le même temps, l'invisibilité infligée aux réfugiés dans le temps où l'on prétend les contrôler, se retourne en processus d'échappement à l'instance de contrôle elle-même.

53 Ainsi, un livre récent de l'anthropologue Michel Agier interroge les nouveaux positionnements des réfugiés face au "gouvernement humanitaire " qui s'érige en instance globale non seulement de contrôle et de normalisation, mais même de répression, puisqu'il mentionne les épisodes réitérés d'appels du HCR à la police, ouvrant le feu contre les réfugiés birmans en Inde, ou contre les réfugiés soudanais en Égypte :

Les camps de réfugiés ne sont pas des zones de non-droit, mais des zones de droit et de pouvoir d'exception, où tout parait possible pour qui les contrôle ${ }^{3}$.

Dans le même temps où les instances biopolitiques de contrôle international organisent, en tant qu'agences humanitaires, le tri et la mise en catégories nosographiques des sujets en vue de les réduire au statut de "populations déplacées ", 
ces mêmes sujets, transgressant le statut auquel les assigne leur vulnérabilité pour le transmuer en revendication, exigent d'être reconnus comme acteurs politiques en opposition même aux puissances « bienfaitrices » qui les tiennent en tutelle.

Si donc les migrations sont bien au cœur des mutations géopolitiques contemporaines, ce n'est pas seulement parce qu'elles modifient, insidieusement autant que massivement, le paysage humain, mais aussi parce qu'elles offrent un nouvel angle d'attaque au retournement polémique de l'assujettissement en subjectivation. De ce point de vue, l'expérience même des migrations apparaît comme la matrice d'une nouvelle pensée du politique, pour laquelle la sédentarité offre de moins en moins la possibilité non seulement de penser l'espace public, mais de penser l'idée même d'humanité.

\section{BIBLIOGRAPHIE}

Agier Michel, Gérer les indésirables, Paris, Flammarion, 2008.

Bauman Zygmunt, Le Présent liquide, peurs sociales et obsession sécuritaire, Paris, Seuil, 2007.

Foucault Michel, Dits et écrits, Paris, Gallimard, 2001.

\section{NOTES}

1. Zygmunt Bauman, Le Présent liquide, peurs sociales et obsession sécuritaire, Paris, Seuil, 2007, p. 7.

2. Michel Foucault, Dits et écrits, Paris, Gallimard, 2001, p. 1266.

3. Michel Agier, Gérer les indésirables, Paris, Flammarion, 2008, p. 128.

\section{RÉSUMÉS}

Ce travail s'appuiera sur le concept d'espace tel qu'il se fonde dans l'Esthétique transcendantale chez Kant (comme condition interne au sujet de toute sa relation au monde), pour montrer en quoi les politiques contemporaines de spatialisation constituent un véritable déni infligé aux possibilités élémentaires de représentation de soi.

Ainsi des processus de désesthétisation sont-ils liés à cette contradiction radicale : un effacement des frontières dans la globalisation des circuits financiers et des réseaux de communication, dans le temps même d'une intensification des frontières comme obstacles à la circulation des personnes. La modernité des spatialisations économiques s'y affronte à un archaïsme des représentations de l'espace national. 
Un travail de terrain, effectué dans des centres de réfugiés tchétchènes en Pologne, met en évidence les différents niveaux de ce régime de duplicité, inscrivant le biopolitique non pas seulement dans la perspective classiquement foucaldienne d'un régime de contrôle, mais dans les contradictions internes d'un contrôle accru et d'une dérégulation. Sans cesse, les migrants sont confrontés à cette duplicité juridique et politique qui fait de la « régulation des flux » une pure et simple amorce de la disparition des personnes. Les régimes de duplicité contemporains, en usant du double langage juridique pour euphémiser la réalité de la violence, sont donc au fondement d'une véritable perversion du politique.

Et cette duplicité mondialisée s'avère, pour les migrants, véritablement terrorisante : livrant les sujets au double langage de l'abandon et de la protection, elle laisse en suspens au-dessus d'eux une menace, implicite et au sens propre affolante, d'extermination.

\section{AUTEUR}

\section{CHRISTIANE VOLLAIRE}

Philosophe 